\title{
CAPACIDADE DE COMBINAÇÃO DE LINHAGENS ELITE DE PIMENTÃO (Capsicum annuum L.)
}

\author{
Combining ability among lines of sweet pepper (Capsicum annuum L.)
}

\author{
Márcia Lídia Gomide', Wilson Roberto Maluf ${ }^{2}$, Luiz Antonio Augusto Gomes ${ }^{3}$
}

\begin{abstract}
RESUMO
Conduziu-se este trabalho, com o objetivo de avaliar a capacidade de combinação de linhagens elite de pimentão (Capisicum annиит L.), identificar combinações híbridas superiores quanto à qualidade de frutos, e inferir sobre os modos de ação gênica envolvidos na expressão dos caracteres relacionados à qualidade dos frutos. Para isto, foram realizados cruzamentos dialélicos para obtenção de híbridos, sendo analisados segundo o esquema dialélico parcial tipo North Carolina II, estimando-se a capacidade geral (CGC) de cada genitor dentro de cada grupo, bem como as capacidades específicas (CEC) de combinação para cada combinação de genitores. Os híbridos experimentais apresentaram heterose para diâmetro de fruto e profundidade de inserção de pedúnculo. A característica profundidade de inserção do pedúnculo é condicionada, predominantemente, por alelos recessivos. Os melhores híbridos, com perspectivas para uso comercial foram $\mathrm{F}_{1}$ (L-3436 x L-004), $\mathrm{F}_{1}$ (L-3513 x L-004) e $\mathrm{F}_{1}$ (L-3509 x L-004). Foram obtidos valores positivos de heterose padrão variando de 1,47 a $30,04 \%$ para diâmetro de fruto, de 2,57 a $83,71 \%$ para profundidade de inserção do pedúnculo, em relação à cultivar híbrida $\mathrm{F}_{1}$ Magali-R.
\end{abstract}

Termos para indexação: Heterose, pimentão, híbridação, capacidade combinatória, Capsicum annuum.

\begin{abstract}
The work had the aim to evaluating the combining ability among sweet pepper breeding lines, identify superior hybrids for fruit quality, and to infer on the mode of gene action involved in the expression fruit quality. North Carolina-II scheme partial diallel crosses were obtained, to estimate general combining abilities (GCA) of parental lines and specific combining abilities (SCA) of parental combinations. There was evidence for heterosis among experimental hybrids for fruit diameter and insertion depth of the peduncle. Peduncle insertion depth is conditioned, predominantly, by recessive alleles. Experimental hybrids with commercial potential were $\mathrm{F}_{1}$ (L-3436 x L-004), $\mathrm{F}_{1}$ (L-3513 x L-004) and $\mathrm{F}_{1}$ (L-3509 x L-004). Heterosis values relative to the standard cultivar Magali-R-F $\mathrm{F}_{1}$ ranged from $1.47 \%$ to $30.04 \%$ for fruit diameter and $2.57 \%$ to $83.71 \%$ for insertion depth of the peduncle.
\end{abstract}

Index terms: Heterosis, sweet pepper, hybrids, combining abilities, Capsicum annuum.

(Recebido em 3 de janeiro de 2005 e aprovado em 21 de janeiro de 2008)

\section{INTRODUÇÃO}

Devido à ampla diversidade genética existente entre espécies do gênero Capsicum é possível a utilização do germoplasma do pimentão (Capsicum annuum L.) em programas de melhoramento (DESHPANDE et al., 1988). Nos últimos anos tem sido dada ênfase à obtenção de híbridos F $_{1}$ (GALVÊAS, 1988; INNECCO, 1995; MIRANDA, 1987; TAVARES, 1993), que podem ser obtidos por meio do cruzamento de linhagens (IKUTA, 1971), resultando em pimentões mais produtivos que as cultivares comerciais de polinização aberta. Prova disto, é que a partir do final da década de 1980 surgiram os primeiros híbridos comerciais desenvolvidos por empresas de sementes instaladas no Brasil. Daquela época até os dias de hoje foi possível verificar um incremento substancial nas áreas de plantio com cultivares híbridas, onde somente no estado de Minas Gerais, que representa 17,6\% do total cultivado no Brasil, as cultivares híbridas ocupam 99\% da área (BLAT, 1999). De acordo com Echer (2001) é estimado um mercado de 2 milhões de dólares somente para o segmento de sementes híbridas dessa hortaliça no Brasil.

O uso de híbridos é vantajoso porque podem combinar no híbrido $\mathrm{F}_{1}$ caracteres importantes presentes em duas linhagens contrastantes. Essa vantagem é ampliada pelo benefício da heterose em características importantes como produtividade, qualidade e uniformidade. Em geral, na obtenção de híbridos de espécies autógamas, como o pimentão, adotam-se esquemas de híbridos simples, pois as linhagens homozigóticas não perdem vigor, não afetando, portanto, a produção de sementes (MELO, 1997).

\footnotetext{
'Bióloga, Doutora em Genética e Melhoramento de Plantas - Departamento de Entomologia/DEN - Universidade Federal de Lavras - Cx. P. 3037 37200-000 - Lavras, MG - marcia_gomide@yahoo.com.br

${ }^{2}$ PhD., Professor - Departamento de Agricultura/DAG - Universidade Federal de Lavras - Cx. P. 3037 - 37200-000 - Lavras, MG - wrmaluf@ufla.br ${ }^{3}$ Engenheiro Agrônomo, Doutor - Departamento de Agricultura/DAG - Universidade Federal de Lavras - Cx. P. 3037 - $37200-000$ - Lavras, MG laagomes@ufla.br
} 
Neste trabalho, objetivou-se avaliar a capacidade combinatória de linhagens elite de pimentão no que diz respeito à características dos frutos, identificar combinações híbridas superiores quanto à qualidade de frutos, e inferir sobre os modos de ação gênica envolvidos na expressão dos caracteres relacionados à qualidade dos frutos.

\section{MATERIAL E MÉTODOS}

O experimento foi conduzido na Estação de Pesquisa de Hortaliças da HortiAgro Sementes Ltda, localizada no município de Ijaci-MG. Dois grupos de linhagens ou cultivares foram utilizados como genitores na obtenção de híbridos experimentais para análise dialélica. O primeiro grupo compreendeu 14 linhagens ou cultivares (1. Agronômico-8; 2. Hércules; 3. Ikeda; 4. Itapetininga; 5. L005; 6. L-3436; 7. L-3509; 8. L-3513; 9. Magda; 10. PIX021E0818; 11. PIX-021E1235; 12. PIX-021E1545; 13. PIX022E31; 14. PIX-023D09), todas utilizadas como genitores femininos. O segundo grupo compreendeu duas linhagens (1'. L-004; 2'. L-006) utilizadas como genitores masculinos e testadores da capacidade de combinação das linhagens do primeiro grupo.

O delineamento experimental utilizado foi o de blocos casualizados completos com 38 tratamentos e três repetições.

Os híbridos experimentais foram analisados segundo o esquema dialélico parcial tipo North Carolina II. Para cada característica estudada, foi calculada a razão (R) entre a variância genética dos híbridos envolvendo o genitor L-004 e a variância genética dos híbridos do genitor L-006. Foram avaliadas as seguintes características: Comprimento de fruto $(\mathbf{m m})=15$ frutos por parcela foram medidos no seu comprimento longitudinal, utilizando-se a média para representar cada parcela; Diâmetro de fruto $(\mathbf{m m})=15$ frutos por parcela foram medidos no seu terço superior, utilizando-se a média para representar cada parcela; Relação comprimento/diâmetro (C/D) de fruto: foi calculada por meio dos valores de comprimento pela largura de cada fruto individualmente; Profundidade de inserção do pedúnculo do fruto (PIPF) (cm): mediu-se a profundidade de inserção do pedúnculo do fruto dos 15 frutos amostrados de cada parcela, utilizando-se a média para representar cada parcela.

\section{RESULTADOS E DISCUSSÃO}

\section{a) Comprimento de fruto:}

Os materiais que produziram, em média, frutos mais compridos, foram Atenas $\mathrm{F}_{1}(164,978 \mathrm{~mm})$, Magali-R $\mathrm{F}_{1}$
(164,267 mm) e Magali- $F_{1}(163,156 \mathrm{~mm})$, e dentre os híbridos experimentais foram $\mathrm{F}_{1}(\mathrm{~L}-3513 \mathrm{x} \mathrm{L}-004)(153,733 \mathrm{~mm}), \mathrm{F}_{1}(\mathrm{~L}-$ 3436 x L-006) (150,711 mm) e $F_{1}$ (Ikeda x L-004) (149,889 $\mathrm{mm}$ ), enquanto $\mathrm{F}_{1}$ (Agronômico-8 x L-006), $\mathrm{F}_{1}$ (L-005 x L006) e $\mathrm{F}_{1}$ (PIX-021E1235 x L-006) produziram os frutos mais curtos (Tabela 1).

As testemunhas de polinização aberta tiveram frutos apenas 2,338 mm, em média, mais curtos do que os 30 híbridos experimentais, valor não significativo e que pode refletir a pouca importância da heterose e dos efeitos gênicos não aditivos, para comprimento de frutos (Tabela 2).

Frutos com maiores comprimentos foram obtidos, em média, quando se utilizou como genitor a linhagem L004 (A) ao invés da linhagem L-006 (B) (Tabela 2). No entanto, como o valor da relação $\mathrm{A} / \mathrm{B}$ é próximo de 1 , ambos os testadores A e B possuem freqüências semelhantes de alelos dominantes para comprimento do fruto, ou seja, ambos se equivalem como testadores para essa característica (Tabela 3). É possível que o valor A/B próximo de 1 também reflita a pouca importância dos efeitos gênicos não aditivos para essa característica.

Resultados diferentes foram obtidos por Blank (1997), que verificou que essa característica deve ser controlada por alelos dominantes no sentido de frutos mais compridos e que a linhagem L-004 possui alelos predominantemente dominantes para as características comprimento de fruto e relação $\mathrm{C} / \mathrm{D}$, e alelos recessivos para a característica diâmetro de fruto.

O efeito da CGC para comprimento de fruto foi significativo somente para alguns genitores do grupo I (Tabela 2). Não se observaram valores significativos para efeitos da CEC dessa característica (Tabela 2). Isso indica que não houve desvios dos híbridos em relação ao que seria esperado com base na CGC dos seus parentais (Tabela 2) e que os efeitos gênicos não aditivos são inexistentes ou irrelevantes para esta característica. Evidencia-se, portanto, a grande importância dos efeitos gênicos aditivos na expressão do comprimento, indicando que o comportamento médio dos híbridos pode ser previsto pela performance média dos seus genitores. Resultados semelhantes foram obtidos por Tavares (1993).

As linhagens parentais que apresentaram os valores mais altos da estimativa de CGC, para comprimento de fruto foram L-3513 e L-3436 e a que apresentou o valor mais baixo foi PIX-021E1235. Híbridos com as linhagens parentais L-3513 e L-3436 produziram frutos com cerca de $9 \mathrm{~mm}$ mais compridos do que a média do grupo I (Tabela 4). Por outro lado, híbridos com a linhagem parental PIX$021 \mathrm{E} 1235$ tiveram a média do comprimento do fruto reduzida em $11,49 \mathrm{~mm}$. 
Tabela 1 - Médias de comprimento e diâmetro de fruto $(\mathrm{mm})$, relação comprimento/diâmetro e profundidade de inserção do pedúnculo (cm) de pimentão, por tratamento. UFLA, Lavras - MG, 2001.

\begin{tabular}{|c|c|c|c|c|}
\hline Tratamento & $\begin{array}{l}\text { Comprimento do } \\
\text { fruto }\end{array}$ & $\begin{array}{l}\text { Diâmetro do } \\
\text { fruto }\end{array}$ & $\begin{array}{c}\text { Relação } \\
\text { Comp/Diâm }\end{array}$ & $\begin{array}{c}\text { Profundidade do } \\
\text { pedúnculo }\end{array}$ \\
\hline 1 - Acauã & $149,553 \mathrm{~b}$ & $73,653 \mathrm{~d}$ & $2,033 \mathrm{~b}$ & $1,240 \mathrm{c}$ \\
\hline 2 - Atenas F1 & $164,977 \mathrm{a}$ & $74,863 \mathrm{~d}$ & $2,200 \mathrm{a}$ & $1,560 \mathrm{~b}$ \\
\hline 3 - Fortuna Super F1 & $143,887 \mathrm{c}$ & $73,147 \mathrm{~d}$ & $1,967 \mathrm{~b}$ & $1,230 \mathrm{c}$ \\
\hline 4 - Ikeda & $136,887 \mathrm{~d}$ & $68,390 \mathrm{f}$ & $2,000 \mathrm{~b}$ & $0,760 \mathrm{e}$ \\
\hline 5 - Magali-R F1 & $164,267 \mathrm{a}$ & $70,297 \mathrm{e}$ & $2,337 \mathrm{a}$ & $1,167 \mathrm{c}$ \\
\hline 6 - Magali F1 & $163,157 \mathrm{a}$ & $71,560 \mathrm{e}$ & $2,280 \mathrm{a}$ & $1,260 \mathrm{c}$ \\
\hline 7 - Magda & $139,023 \mathrm{c}$ & $67,567 \mathrm{f}$ & $2,067 \mathrm{~b}$ & $0,627 \mathrm{e}$ \\
\hline $8-\operatorname{Myr} 29$ & $148,777 \mathrm{~b}$ & $69,713 \mathrm{e}$ & $2,133 \mathrm{~b}$ & $0,987 \mathrm{~d}$ \\
\hline 9 - F1 (L 006 x L 004) & $134,580 \mathrm{~d}$ & 91,413 a & $1,477 \mathrm{~d}$ & $1,637 \mathrm{~b}$ \\
\hline 10 - F1 (PIX 025 Hms x L 004) & $146,200 \mathrm{~b}$ & $76,167 \mathrm{c}$ & $1,920 \mathrm{c}$ & $1,577 \mathrm{~b}$ \\
\hline 11 - F1 (Agronômico 8 x L 004) & $139,600 \mathrm{c}$ & $77,987 \mathrm{c}$ & $1,793 \mathrm{c}$ & $1,997 \mathrm{a}$ \\
\hline 12 - F1 (Agronômico 8 x L 006) & $132,333 \mathrm{~d}$ & $65,433 \mathrm{f}$ & $2,023 \mathrm{~b}$ & $1,243 \mathrm{c}$ \\
\hline 13 - F1 (Hércules x L 004) & $143,467 \mathrm{c}$ & $72,813 \mathrm{~d}$ & $1,970 \mathrm{~b}$ & $1,097 \mathrm{c}$ \\
\hline 14 - F1 (Hércules x L 006) & $135,620 \mathrm{~d}$ & $73,027 \mathrm{~d}$ & $1,863 \mathrm{c}$ & $0,983 \mathrm{~d}$ \\
\hline 15 - F1 (Ikeda x L 004) & $149,887 \mathrm{~b}$ & $74,890 \mathrm{~d}$ & $2,003 \mathrm{~b}$ & $1,450 \mathrm{c}$ \\
\hline 16 - F1 (Ikeda x L 006) & $136,380 \mathrm{~d}$ & $74,343 \mathrm{~d}$ & $1,833 \mathrm{c}$ & $0,947 \mathrm{~d}$ \\
\hline 17 - F1 (Itapetininga x L 004) & $146,290 \mathrm{~b}$ & $79,647 \mathrm{c}$ & $1,837 \mathrm{c}$ & $1,907 \mathrm{a}$ \\
\hline 18 - F1 (Itapetininga x L 006) & $147,177 \mathrm{~b}$ & $74,280 \mathrm{~d}$ & $1,980 \mathrm{~b}$ & $1,197 \mathrm{c}$ \\
\hline $19-\mathrm{F} 1(\mathrm{~L} 005$ x L 004) & $140,823 \mathrm{c}$ & 90,470 a & $1,557 \mathrm{~d}$ & $2,033 \mathrm{a}$ \\
\hline $20-\mathrm{F} 1$ (L 005 x L 006) & $130,443 \mathrm{~d}$ & 88,207 a & $1,483 \mathrm{~d}$ & $1,587 \mathrm{~b}$ \\
\hline $21-\mathrm{F} 1$ (L 3436 x L 004) & $149,867 \mathrm{~b}$ & $78,187 \mathrm{c}$ & $1,920 \mathrm{c}$ & $1,773 \mathrm{~b}$ \\
\hline $22-\mathrm{F} 1$ (L 3436 x L 006) & $150,713 \mathrm{~b}$ & $78,620 \mathrm{c}$ & $1,923 \mathrm{c}$ & $1,283 \mathrm{c}$ \\
\hline 23 - F1 (L 3509 x L 004) & $148,510 \mathrm{~b}$ & $86,273 \mathrm{a}$ & $1,723 \mathrm{c}$ & $2,103 \mathrm{a}$ \\
\hline $24-F 1$ (L 3509 x L 006) & $143,777 \mathrm{c}$ & $78,280 \mathrm{c}$ & $1,847 \mathrm{c}$ & $1,240 \mathrm{c}$ \\
\hline $25-\mathrm{F} 1$ (L 3513 x L 004) & $153,733 \mathrm{~b}$ & $83,217 \mathrm{~b}$ & $1,847 \mathrm{c}$ & $2,143 \mathrm{a}$ \\
\hline $26-F 1(L 3513$ x L 006) & $148,333 \mathrm{~b}$ & $80,963 \mathrm{~b}$ & $1,830 \mathrm{c}$ & $1,670 \mathrm{~b}$ \\
\hline 27 - F1 (Magda x L 004) & $135,020 \mathrm{~d}$ & $73,170 \mathrm{~d}$ & $1,847 \mathrm{c}$ & $1,010 \mathrm{~d}$ \\
\hline 28 - F1 (Magda x L 006) & $136,577 \mathrm{~d}$ & $74,317 \mathrm{~d}$ & $1,840 \mathrm{c}$ & $1,110 \mathrm{c}$ \\
\hline 29 - F1 (PIX 021E 0818 x L 004) & $144,490 \mathrm{c}$ & $72,997 \mathrm{~d}$ & $1,980 \mathrm{~b}$ & $0,943 \mathrm{~d}$ \\
\hline 30 - F1 (PIX 021E 0818 x L 006) & $138,180 \mathrm{c}$ & $75,317 \mathrm{~d}$ & $1,837 \mathrm{c}$ & $1,137 \mathrm{c}$ \\
\hline 31 - F1 (PIX 021E 1235 x L 004) & $132,667 \mathrm{~d}$ & $76,700 \mathrm{c}$ & $1,730 \mathrm{c}$ & $1,217 \mathrm{c}$ \\
\hline 32 - F1 (PIX 021E 1235 x L 006) & $126,913 \mathrm{~d}$ & $73,403 \mathrm{~d}$ & $1,727 \mathrm{c}$ & $1,260 \mathrm{c}$ \\
\hline 33 - F1 (PIX 021E 1545 x L 004) & $138,670 \mathrm{c}$ & $71,853 \mathrm{e}$ & $1,933 \mathrm{c}$ & $1,363 \mathrm{c}$ \\
\hline 34 - F1 (PIX 021E 1545 x L 006) & $136,467 \mathrm{~d}$ & $71,330 \mathrm{e}$ & $1,917 \mathrm{c}$ & $1,163 \mathrm{c}$ \\
\hline 35 - F1 (PIX 022E 31 x L 004) & $138,220 \mathrm{c}$ & $76,500 \mathrm{c}$ & $1,807 \mathrm{c}$ & $1,590 \mathrm{~b}$ \\
\hline 36 - F1 (PIX 022E 31 x L 006) & $142,713 \mathrm{c}$ & $75,240 \mathrm{~d}$ & $1,900 \mathrm{c}$ & $1,253 \mathrm{c}$ \\
\hline 37 - F1 (PIX 023D 09 x L 004) & $143,577 \mathrm{c}$ & $67,650 \mathrm{f}$ & $2,123 \mathrm{~b}$ & $1,610 \mathrm{~b}$ \\
\hline 38 - F1 (PIX 023D 09 x L 006) & $145,420 \mathrm{~b}$ & $62,977 \mathrm{f}$ & $2,310 \mathrm{a}$ & $1,217 \mathrm{c}$ \\
\hline
\end{tabular}

Médias seguidas pela mesma letra na coluna não diferem entre si (Scott-Knott; $p>0,05)$. 
Tabela 2 - Resumo da análise de variância para as características comprimento e diâmetro de fruto (mm), relação comprimento/diâmetro e profundidade de inserção do pedúnculo (mm). UFLA, Lavras - MG, 2000.

\begin{tabular}{|c|c|c|c|c|c|}
\hline \multirow[b]{2}{*}{ Fontes de Variação } & \multirow[b]{2}{*}{ GL } & \multicolumn{4}{|c|}{ QM } \\
\hline & & $\begin{array}{l}\text { Comprimento de } \\
\text { fruto }(\mathrm{C})\end{array}$ & $\begin{array}{l}\text { Diâmetro de } \\
\text { fruto (D) }\end{array}$ & Relação C/D & Pedúnculo \\
\hline Bloco & 2 & $122,775 *$ & $30,967 *$ & $0,0770 * *$ & 0,092 \\
\hline Tratamentos & 37 & $233,358 * *$ & $119,861 * *$ & $0,114 * *$ & $0,404 * *$ \\
\hline $\begin{array}{l}\text { Entre testemunhas e os } \\
\text { híbridos do dialelo }\end{array}$ & 1 & $1362,017 * *$ & $119,626 * *$ & $0,643 * *$ & $0,913 * *$ \\
\hline Entre testemunhas & 9 & $392,370 * *$ & $139,791 * *$ & $0,173 * *$ & $0,346 * *$ \\
\hline Entre híbridos do dialelo & 27 & $138,552 * *$ & $113,226 * *$ & $0,075 * *$ & $0,405 * *$ \\
\hline Entre genitores femininos & 13 & $222,985 * *$ & $200,284 * *$ & $0,132 * *$ & $0,483 * *$ \\
\hline Entre genitores masculinos & 1 & $309,811 *$ & $143,655 *$ & 0,006 & $2,622 * *$ \\
\hline $\begin{array}{l}\text { Entre genitores femininos } \mathrm{x} \\
\text { genitores masculinos }\end{array}$ & 13 & 40,945 & 23,828 & 0,023 & $0,157 *$ \\
\hline Resíduo & 74 & 33,264 & 9,075 & 0,009 & 0,033 \\
\hline Total & 113 & & & & \\
\hline Contrastes de interesse & & \multicolumn{4}{|c|}{ Estimativa } \\
\hline $\begin{array}{l}\text { Testemunhas F1 vs testemunhas } \\
\text { de polinização aberta }\end{array}$ & 1 & $62,047 * *$ & $10,543 *$ & $0,550 * *$ & $1,603 * *$ \\
\hline $\begin{array}{l}\text { Efeito do alelo ms [F1 } \\
\text { (IkedaxL004) vs F1 (PIX- } \\
\text { 025HmsxL004)] }\end{array}$ & 1 & $-3,687$ & 1,277 & $-0,083$ & 0,127 \\
\hline $\begin{array}{l}\text { Testem. de polinização aberta vs } \\
30 \text { híbridos experimentais }\end{array}$ & 1 & 2,338 & $-6,691 * *$ & $0,199 * *$ & $-0,521 * *$ \\
\hline $\begin{array}{l}\text { Testemunhas-híbridos comerciais } \\
\text { vs } 30 \text { híbridos experimentais }\end{array}$ & 1 & $17,850 * *$ & $-4,055 * *$ & $0,337 * *$ & $-0,121 *$ \\
\hline $\begin{array}{l}\text { Híbridos com genitor L004 vs } \\
\text { híbridos com genitor L006 }\end{array}$ & 1 & $3,841 * *$ & $2,615 * *$ & $-0,017$ & $0,353 * *$ \\
\hline
\end{tabular}

Tabela 3 - Variância genética dos caracteres avaliados nos híbridos com genótipos com L004 e L006 e a relação entre eles. Lavras - MG. 2000.

\begin{tabular}{lccc}
\hline \multirow{2}{*}{ Característica } & \multicolumn{2}{c}{ Variância genética entre híbridos com } \\
\cline { 2 - 4 } & $\begin{array}{c}\text { Genótipo parental } \\
\text { L004 (A) }\end{array}$ & $\begin{array}{c}\text { Genótipo parental } \\
\text { L006 (B) }\end{array}$ & A/B \\
\hline Comprimento do fruto (cm) & 25,952 & 39,848 & 0,651 \\
Diâmetro do fruto (cm) & 33,978 & 34,676 & 0,979 \\
Relação comp/diâm do fruto & 0,017 & 0,0285 & 0,599 \\
Profundidade de Inserção do & 0,164 & 0,0272 & 6,041 \\
pedúnculo (mm) & & & \\
\hline
\end{tabular}

A média dos tratamentos para comprimento de fruto foi de 141,281 e as amplitudes da CGC entre os genitores do grupo I, e da CEC foram, respectivamente, 21,243 e 9,666.
Os valores das amplitudes não são representativos em relação à média, ou seja, os efeitos métricos são pouco marcantes (Tabela 4). 
Tabela 4 - Estimativas da capacidade geral (CGC) de combinação dos genitores femininos e masculinos e capacidade específica de combinação (CEC) dos híbridos entre eles para as características comprimento de fruto (cm), diâmetro de fruto $(\mathrm{mm})$, relação comprimento/diâmetro e profundidade de inserção do pedúnculo (mm). UFLA, Lavras - MG, 2000.

\begin{tabular}{|c|c|c|c|c|}
\hline \multirow{2}{*}{ Parâmetro } & \multicolumn{4}{|c|}{ Estimativa ( \pm erro padrão) } \\
\hline & Comprimento Fruto (C) & Diâmetro Fruto (D) & Relação C/D & Pedúnculo \\
\hline$\mu$ & $141,281( \pm 2,708) * *$ & $76,003( \pm 1,569) *$ & $1,871( \pm 0,051) * *$ & $1,412( \pm 0,086) * *$ \\
\hline \multicolumn{5}{|c|}{ Capacidade geral de combinação (genitores femininos) (gi) } \\
\hline $\mathrm{g}_{1}$ Agronômico 8 & $-5,314( \pm 3,267)$ & $-4,293( \pm 1,893)$ & $0,038( \pm 0,061)$ & $0,208( \pm 0,104) *$ \\
\hline $\mathrm{g}_{2}$ Hércules & $-1,738( \pm 3,267)$ & $-3,083( \pm 1,893)$ & $0,046( \pm 0,061)$ & $-0,372( \pm 0,104) * *$ \\
\hline $\mathrm{g}_{3}$ Ikeda & $1,852( \pm 3,267)$ & $-1,387( \pm 1,893)$ & $0,048( \pm 0,061)$ & $-0,213( \pm 0,104) *$ \\
\hline $\mathrm{g}_{4}$ Itapetininga & $5,452( \pm 3,267)$ & $0,960( \pm 1,893)$ & $0,038( \pm 0,061)$ & $0,140( \pm 0,104)$ \\
\hline $\mathrm{g}_{5} \mathrm{~L}-005$ & $-5,648( \pm 3,267)$ & $13,335( \pm 1,893) * *$ & $-0,351( \pm 0,061) * *$ & $0,398( \pm 0,104) * *$ \\
\hline $\mathrm{g}_{6} \mathrm{~L}-3436$ & $9,009( \pm 3,267) * *$ & $2,400( \pm 1,893)$ & $0,051( \pm 0,061)$ & $0,1167( \pm 0,104)$ \\
\hline $\mathrm{g}_{7} \mathrm{~L}-3509$ & $4,862( \pm 3,267)$ & $6,273( \pm 1,893) * *$ & $-0,086( \pm 0,061)$ & $0,260( \pm 0,104) *$ \\
\hline $\mathrm{g}_{8} \mathrm{~L}-3513$ & $9,752( \pm 3,267) * *$ & $6,087( \pm 1,893) * *$ & $-0,033( \pm 0,061)$ & $0,495( \pm 0,104) * *$ \\
\hline $\mathrm{g}_{9}$ Magda & $-5,483( \pm 3,267)$ & $-2,260( \pm 1,893)$ & $-0,028( \pm 0,061)$ & $-0,352( \pm 0,104) * *$ \\
\hline $\mathrm{g}_{10}$ PIX-021E0818 & $0,054( \pm 3,267)$ & $-1,847( \pm 1,893)$ & $0,038( \pm 0,061)$ & $-0,372( \pm 0,104) * *$ \\
\hline $\mathrm{g}_{11}$ PIX-021E1235 & $-11,491( \pm 3,267)^{* *}$ & $-0,952( \pm 1,893)$ & $-0,143( \pm 0,061) *$ & $-0,173( \pm 0,104)$ \\
\hline $\mathrm{g}_{12}$ PIX-021E1545 & $-3,713( \pm 3,267)$ & $-4,412( \pm 1,893) *$ & $0,054( \pm 0,061)$ & $-0,148( \pm 0,104)$ \\
\hline $\mathrm{g}_{13}$ PIX-022E31 & $-0,814( \pm 3,267)$ & $-0,133( \pm 1,893)$ & $-0,018( \pm 0,061)$ & $0,010( \pm 0,104)$ \\
\hline $\mathrm{g}_{14}$ PIX-023D09 & $3,217( \pm 3,267)$ & $-10,690( \pm 1,893) * *$ & $0,346( \pm 0,061)^{* *}$ & $0,002( \pm 0,104)$ \\
\hline \multicolumn{5}{|c|}{ Capacidade geral de combinação (genitores masculinos) (gj) } \\
\hline $\mathrm{g}_{1}, \mathrm{~L}-004$ & $1,920( \pm 2,684)$ & $1,308( \pm 1,555)$ & $-0,009( \pm 0,050)$ & $0,177( \pm 0,085)^{*}$ \\
\hline $\mathrm{g}_{2} \cdot \mathrm{L}-006$ & $-1,920( \pm 2,684)$ & $-1,308( \pm 1,555)$ & $0,009( \pm 0,050)$ & $-0,177( \pm 0,055)^{*}$ \\
\hline \multicolumn{5}{|c|}{ Capacidade específica de combinação } \\
\hline $\mathrm{s}_{1.1}$ Agronômico-8 x L-004 & $1,713( \pm 3,266)$ & $4,969( \pm 1,892)^{*}$ & $-0,106( \pm 0,061)$ & $0,200( \pm 0,104)$ \\
\hline $\mathrm{s}_{1.2}$, Agronômico-8 x L-006 & $-1,713( \pm 3,266)$ & $-4,969( \pm 1,892) *$ & $0,106( \pm 0,061)$ & $-0,200( \pm 0,104)$ \\
\hline $\mathrm{s}_{2.1}$. Hércules x L-004 & $2,003( \pm 3,266)$ & $-1,414( \pm 1,892)$ & $0,062( \pm 0,061)$ & $-0,120( \pm 0,104)$ \\
\hline $\mathrm{s}_{2.2}$. Hércules x L-006 & $-2,003( \pm 3,266)$ & $1,414( \pm 1,892)$ & $-0,062( \pm 0,061)$ & $0,120( \pm 0,104)$ \\
\hline $\mathrm{s}_{3.1}$, Ikeda x L-004 & $4,833( \pm 3,266)$ & $-1,034( \pm 1,892)$ & $0,094( \pm 0,061)$ & $0,075( \pm 0,104)$ \\
\hline $\mathrm{s}_{3.2}$ Ikeda $\mathrm{x}$ L-006 & $-4,833( \pm 3,266)$ & $1,034( \pm 1,892)$ & $-0,094( \pm 0,061)$ & $-0,075( \pm 0,104)$ \\
\hline $\mathrm{s}_{4.1}$, Itapetininga $\mathrm{x}$ L-004 & $-2,364( \pm 3,266)$ & $1,376( \pm 1,892)$ & $-0,063( \pm 0,061)$ & $0,178( \pm 0,104)$ \\
\hline $\mathrm{s}_{4.2^{\prime}}$ Itapetininga $\mathrm{x}$ L-006 & $2,364( \pm 3,266)$ & $-1,376( \pm 1,892)$ & $0,063( \pm 0,061)$ & $-0,178( \pm 0,104)$ \\
\hline $\mathrm{s}_{5.1}, \mathrm{~L}-005 \times \mathrm{L}-004$ & $3,270( \pm 3,266)$ & $-0,176( \pm 1,892)$ & $0,045( \pm 0,061)$ & $0,047( \pm 0,104)$ \\
\hline$s_{5.2} \cdot$ L-005 x L-006 & $-3,270( \pm 3,266)$ & $0,176( \pm 1,892)$ & $-0,045( \pm 0,061)$ & $-0,047( \pm 0,104)$ \\
\hline$s_{6.1}, L-3436 \times$ L-004 & $-2,344( \pm 3,266)$ & $-1,524( \pm 1,892)$ & $0,007( \pm 0,061)$ & $0,068( \pm 0,104)$ \\
\hline $\mathrm{s}_{6.2} \cdot \mathrm{L}-3436 \times \mathrm{L}-006$ & $2,344( \pm 3,266)$ & $1,524( \pm 1,892)$ & $-0,007( \pm 0,061)$ & $-0,068( \pm 0,104)$ \\
\hline $\mathrm{s}_{7.1}, \mathrm{~L}-3509 \times \mathrm{L}-004$ & $0,446( \pm 3,266)$ & $2,689( \pm 1,892)$ & $-0,053( \pm 0,061)$ & $0,255( \pm 0,104) *$ \\
\hline $\mathrm{s}_{7.2} \cdot \mathrm{L}-3509 \times \mathrm{L}-006$ & $-0,446( \pm 3,266)$ & $-2,689( \pm 1,892)$ & $0,053( \pm 0,061)$ & $-0,255( \pm 0,104) *$ \\
\hline $\mathrm{s}_{8.1}, \mathrm{~L}-3513 \times \mathrm{L}-004$ & $0,780( \pm 3,266)$ & $-0,181( \pm 1,892)$ & $0,017( \pm 0,061)$ & $0,060( \pm 0,104)$ \\
\hline $\mathrm{s}_{8.2} \cdot \mathrm{L}-3513 \times \mathrm{L}-006$ & $-0,780( \pm 3,266)$ & $0,181( \pm 1,892)$ & $-0,017( \pm 0,061)$ & $-0,060( \pm 0,104)$ \\
\hline $\mathrm{s}_{9.1}$, Magda x L-004 & $-2,699( \pm 3,266)$ & $-1,881( \pm 1,892)$ & $0,012( \pm 0,061)$ & $-0,227( \pm 0,104) *$ \\
\hline $\mathrm{s}_{9.2} \cdot$ Magda x L-006 & $2,699( \pm 3,266)$ & $1,881( \pm 1,892)$ & $-0,012( \pm 0,061)$ & $0,227( \pm 0,104) *$ \\
\hline $\mathrm{s}_{10.1^{\prime}}$ PIX-021E0818 x L-004 & $1,235( \pm 3,266)$ & $-2,468( \pm 1,892)$ & $0,080( \pm 0,061)$ & $-0,273( \pm 0,104) *$ \\
\hline $\mathrm{s}_{10.2^{\prime}}$ PIX-021E0818 x L-006 & $-1,235( \pm 3,266)$ & $2,468( \pm 1,892)$ & $-0,080( \pm 0,061)$ & $0,273( \pm 0,104) *$ \\
\hline $\mathrm{s}_{11.1}$ PIX-021E1235 x L-004 & $0,956( \pm 3,266)$ & $0,341( \pm 1,892)$ & $0,010( \pm 0,061)$ & $-0,198( \pm 0,104)$ \\
\hline $\mathrm{s}_{11.2^{\prime}}$ PIX-021E1235 x L-006 & $-0,956( \pm 3,266)$ & $-0,341( \pm 1,892)$ & $-0,010( \pm 0,061)$ & $0,198( \pm 0,104)$ \\
\hline
\end{tabular}

Continuação... 
Tabela 4 - Continuação...

\begin{tabular}{lrrrr}
\hline $\mathrm{s}_{12.1^{\prime}}$ PIX-021E1545 x L-004 & $-0,819( \pm 3,266)$ & $-1,046( \pm 1,892)$ & $0,017( \pm 0,061)$ & $-0,077( \pm 0,104)$ \\
$\mathrm{s}_{12.2^{\prime}}$ PIX-021E1545 x L-006 & $0,819( \pm 3,266)$ & $1,046( \pm 1,892)$ & $-0,017( \pm 0,061)$ & $0,077( \pm 0,104)$ \\
$\mathrm{s}_{13.1^{\prime}}$ PIX-022E31 x L-004 & $-4,167( \pm 3,266)$ & $-0,678( \pm 1,892)$ & $-0,038( \pm 0,061)$ & $-0,008( \pm 0,104)$ \\
$\mathrm{s}_{13.2^{\prime}}$ PIX-022E31 x L-006 & $4,167( \pm 3,266)$ & $0,678( \pm 1,892)$ & $0,038( \pm 0,061)$ & $0,008( \pm 0,104)$ \\
$\mathrm{s}_{14.1}$ PIX-023D09 x L-004 & $-2,842( \pm 3,266)$ & $1,029( \pm 1,892)$ & $-0,085( \pm 0,061)$ & $0,020( \pm 0,104)$ \\
$\mathrm{s}_{14.2^{\prime}}$ PIX-023D09 x L-006 & $2,842( \pm 3,266)$ & $-1,029( \pm 1,892)$ & $0,085( \pm 0,061)$ & $-0,020( \pm 0,104)$ \\
\hline
\end{tabular}

\section{b) Diâmetro de fruto:}

Frutos com maiores diâmetros foram produzidos por $\mathrm{F}_{1}\left(\mathrm{~L}-006\right.$ x L-004) $(91,415 \mathrm{~mm}), \mathrm{F}_{1}$ (L-005 x L-004) $(90,469 \mathrm{~mm}), \mathrm{F}_{1}$ (L-005 x L-006) (88,201 mm) e $\mathrm{F}_{1}(\mathrm{~L}-3509$ x L-004) (86,273 mm), enquanto Ikeda, $\mathrm{F}_{1}$ (PIX-023D09 x L004), Magda, $F_{1}$ (Agronômico-8 x L-006) e $F_{1}$ (PIX-023D09 $\mathrm{x}$ L-006) produziram frutos com os menores diâmetros (Tabela 1).

Os 30 híbridos experimentais produziram, em média, frutos com diâmetros $6,69 \mathrm{~mm}$ maiores que as testemunhas de polinização aberta, indicando a ocorrência de heterose positiva para essa característica, e de 4,05 $\mathrm{mm}$ maior do que as testemunhas dos híbridos comerciais, indicando a superioridade dos 30 híbridos experimentais em relação aos híbridos comerciais (Tabela 2).

Em comparação com a cultivar padrão Magali-R $\mathrm{F}_{1}$, a característica diâmetro de fruto apresentou heterose padrão positiva variando de 1,47 a $30,04 \%$, sendo que os maiores valores foram obtidos pelos híbridos $\mathrm{F}_{1}$ (L005 x L-006) (25,48\%), F (L-005 x L-004) $(28,70 \%)$ e F 1 (L006 x L-004) (30,04\%) (Tabela 5). Frutos com maiores diâmetros foram obtidos, em média, quando se utilizaram híbridos com genitor L-004 (A) do que com genitor L006 (B) (Tabela 2). No entanto, o valor da relação A/B é próximo de 1 (Tabela 3 ), indicando que ambos os testadores A e B possuem freqüências semelhantes de alelos dominantes para a característica, ou seja, ambos se equivalem como testadores para a mesma. É possível, pois, que a heterose para diâmetro de frutos seja em conseqüência dos efeitos gênicos epistáticos, e não apenas a efeitos de dominância. Resultados discordantes foram obtidos por Blank (1997).

O efeito da CGC foi significativo para a característica diâmetro de fruto, não se observando valores significativos de CEC (Tabela 2). A grande evidência dos efeitos gênicos aditivos na expressão dessa característica mostra que a média das linhagens parentais "per se" indica o comportamento médio dos híbridos para a mesma, embora também se tenha sugerido a ocorrência de efeitos epistáticos, de natureza não aditiva. Resultados concordantes foram obtidos por Tavares (1993).

A linhagem parental L-005 foi a de melhor CGC para diâmetro de fruto, seguida por L-3509 e L-3513, enquanto PIX-023D09 foi a pior, seguida de PIX-021E1545 (Tabela 4). Assim, híbridos com as linhagens parentais L-3513, L-005, L-3509 tiveram aumento na média do diâmetro dos frutos de 6,$08 ; 13,33$ e $6,27 \mathrm{~mm}$, respectivamente; enquanto que os híbridos com os parentais PIX-021E1545 e PIX-023D09 tiveram uma redução do diâmetro do fruto em 4,41 e 10,68 $\mathrm{mm}$, respectivamente (Tabela 4).

Embora o valor de CEC (Tabela 2) não tenha sido significativo, observou-se que o diâmetro do fruto foi influenciado pelas combinações híbridas Agronômico $8 \mathrm{x}$ L-004 e Agronômico-8 x L-006 (Tabela 4).

O maior valor de CGC para diâmetro de fruto foi de 13,335 e o menor de $-10,690$, resultando numa amplitude de 24,025, que é bastante representativa em relação à média $(\mathrm{m}=76,003)$, tendo uma influência substancial na expressão dessa característica (Tabela 4). A amplitude da CEC foi de 9,938 que é pouco representativa em relação à média (Tabela 4). Fica evidente a importância dos efeitos de CGC na expressão dessa característica, embora em alguns casos os valores de CEC também tenham sido importantes.

\section{c) Relação comprimento/diâmetro de fruto:}

A relação comprimento/diâmetro é indicativa do formato do fruto, ou seja, quanto maior o valor da relação $\mathrm{C} / \mathrm{D}$, mais alongados são os frutos. Frutos com maiores médias da relação $\mathrm{C} / \mathrm{D}$ (frutos mais alongados) foram produzidos por Magali R-F $(2,336 \mathrm{~mm}), \mathrm{F}_{1}$ (PIX-023D09 x L-006) (2,312 mm), Magali $F_{1}(2,282 \mathrm{~mm})$ e Atenas $F_{1}(2,201$ $\mathrm{mm}$ ) e os que tiveram as menores relações $\mathrm{C} / \mathrm{D}$ (frutos menos alongados) foram $\mathrm{F}_{1}$ (L-005 x L-004) (1,558 mm), $\mathrm{F}_{1}$ (L-005 x L-006) (1,483 mm) e F 1 (L-006 x L-004) (1,474 mm) (Tabela 1). 
Os 30 híbridos experimentais apresentaram relação C/D de 0,19 unidade a menos que a das testemunhas de polinização aberta, sugerindo, a ocorrência de heterose negativa para essa característica (Tabela 2). Somente os efeitos de CGC entre os genitores do grupo I foram significativos para a relação comprimento/diâmetro (Tabela 2). A grande importância do efeito gênico aditivo na expressão dessa característica é refletida na não-significância da CEC, indicando que a performance média "per se" dos genitores é um bom indicativo da performance média dos híbridos para essa característica. Resultados semelhantes foram obtidos por Tavares (1993).

A relação comprimento/diâmetro foi influenciada significativamente apenas pelas linhagens, do grupo I, L-005 e PIX-021E1235 (negativamente) e PIX-023D09 (positivamente) (Tabela 4). A linhagem parental com maior CGC para a relação C/D foi a PIX-023D09 e a com menor CGC foi L-005, seguida de PIX-021E1235 (Tabela 4). Portanto, híbridos com a linhagem parental PIX023D09 tendem a produzir frutos mais compridos e híbridos com L-005, PIX-021E1235 tendem a produzir frutos mais curtos.

A característica relação C/D apresentou amplitude da CGC, entre os genitores do grupo I, e da CEC, de 0,697 e 0,212 , respectivamente, que são bastante representativas em relação à média $(\mathrm{m}=1,871)$, ou seja, influenciam na expressão dessa característica (Tabela 4).

\section{d) Profundidade de inserção do pedúnculo:}

Frutos com o pedúnculo inserido mais profundamente (característica indesejável) foram produzidos por $\mathrm{F}_{1}$ (L-3513 x L-004) $(2,14 \mathrm{~cm}), \mathrm{F}_{1}$ (L-3509 x L-004) $(2,10 \mathrm{~cm}), \mathrm{F}_{1}\left(\mathrm{~L}-005 \mathrm{x}\right.$ L-004) $(2,03 \mathrm{~cm}), \mathrm{F}_{1}$ (Agronômico-8 x L-004) (1,99 cm) e $\mathrm{F}_{1}$ (Itapetininga x L004) $(1,90 \mathrm{~cm})$, enquanto Ikeda $(0,76 \mathrm{~cm})$ e Magda $(0,63$ $\mathrm{cm})$ produziram frutos com pedúnculo inserido mais superficialmente. Frutos com as maiores médias para a profundidade de inserção do pedúnculo foram obtidos do cruzamento com a linhagem testadora L-004 do que com L-006 (Tabela 1). Portanto, deve-se selecionar os híbridos que apresentaram valores baixos de heterose padrão como, por exemplo, $\mathrm{F}_{1}$ (PIX-021E0818 x L-004) (19,15\%) (Tabela 5).

As testemunhas $\mathrm{F}_{1}$ (híbridos comerciais) são, em média, de inserção mais profunda que as testemunhas de polinização aberta, sugerindo a ocorrência de heterose positiva para essa característica (Tabela 2). A profundidade de inserção do pedúnculo apresentou heterose, em relação a cultivar padrão Magali-R $\mathrm{F}_{1}$, variando de 2,57 a 83,71\% (Tabela 5). No entanto, não são vantajosos valores altos e positivos de heterose para essa característica, porque indica que o pedúnculo está mais profundamente inserido, o que não é desejável.

Pedúnculos mais profundamente inseridos foram, em média, apresentados pelos híbridos com genitor L-004 do que com genitor L-006 (Tabela 2). O valor da relação A/ B é maior que $1(6,0409)$ (Tabela 3$)$, demonstrando que o testador A (L-004), com maior profundidade de inserção do pedúnculo, tem uma frequiência maior de alelos recessivos para essa característica, ou seja, A é o melhor testador para a mesma. Os alelos que controlam maior profundidade de inserção do pedúnculo são, em geral, recessivos.

A característica profundidade de inserção do pedúnculo apresentou valores significativos de CGC entre os genitores do grupo I e II, e valores significativos de CEC (Tabela 2). A maioria das linhagens parentais do grupo I e todas do grupo II apresentaram valores significativos de CGC. Para algumas combinações híbridas, valores de CEC para a característica profundidade de inserção do pedúnculo foram também significativos (Tabela 4). Evidencia-se, portanto, a importância dos efeitos gênicos não aditivos na expressão dessa característica, mostrando que a performance média "per se" dos genitores não é um bom parâmetro para indicar o comportamento médio dos híbridos para a característica em questão.

É desejável que a profundidade de inserção do pedúnculo seja a menor possível, para evitar o acúmulo de água e posterior aparecimento de doenças. Portanto, os piores pais (com maiores valores de CGC), dentro do grupo I, foram L-3513, L-005, L-3509 e Agronômico-8 e os melhores (com menores CGC) foram Hércules, PIX021E0818, Magda e Ikeda. Dentro do grupo II, a linhagem parental com menor CGC foi L-006 e a com maior CGC foi L-004 (Tabela 4).

A profundidade de inserção do pedúnculo apresentou média dos tratamentos de 1,412 e amplitude de CGC, entre os genitores do grupo I, e de CEC, de 0,867 e 0,546 , respectivamente, indicando que a média é influenciada pelos valores de CGC e CEC (Tabela 4). 
Tabela 5 - Heterose padrão (\%) de híbridos de pimentão, obtida em função do híbrido Magali-R $\mathrm{F}_{1}$, para as características comprimento e diâmetro do fruto, relação comprimento/diâmetro e profundidade de inserção do pedúnculo. Lavras MG, 2000.

\begin{tabular}{lcccc}
\hline \multicolumn{1}{c}{ Híbridos de pimentão } & $\begin{array}{c}\text { Comprimento do } \\
\text { fruto }\end{array}$ & $\begin{array}{c}\text { Diâmetro do } \\
\text { fruto }\end{array}$ & $\begin{array}{c}\text { Relação } \\
\text { Comp/Diâm }\end{array}$ & $\begin{array}{c}\text { Profundidade do } \\
\text { pedúnculo }\end{array}$ \\
\hline $\mathrm{F}_{1}$ (L-006 x L-004) & $-18,07$ & 30,04 & $-36,80$ & 40,28 \\
$\mathrm{~F}_{1}$ (PIX-025Hms x L-004) & $-11,00$ & 8,35 & $-17,83$ & 35,14 \\
$\mathrm{~F}_{1}$ (Agronômico-8 x L-004) & $-15,02$ & 10,94 & $-23,25$ & 71,14 \\
$\mathrm{~F}_{1}$ (Agronômico-8 x L-006) & $-19,44$ & $-6,92$ & $-13,41$ & 6,57 \\
$\mathrm{~F}_{1}$ (Hércules x L-004) & $-12,66$ & 3,58 & $-15,69$ & $-6,00$ \\
$\mathrm{~F}_{1}$ (Hércules x L-006) & $-17,44$ & 3,88 & $-20,26$ & $-15,72$ \\
$\mathrm{~F}_{1}$ (Ikeda x L-004) & $-8,75$ & 6,53 & $-14,27$ & 24,28 \\
$\mathrm{~F}_{1}$ (Ikeda x L-006) & $-16,98$ & 5,76 & $-21,54$ & $-18,86$ \\
$\mathrm{~F}_{1}$ (Itapetininga x L-004) & $-10,94$ & 13,30 & $-21,40$ & 63,43 \\
$\mathrm{~F}_{1}$ (Itapetininga x L-006) & $-10,40$ & 5,67 & $-15,26$ & 2,57 \\
$\mathrm{~F}_{1}$ (L-005 x L-004) & $-14,27$ & 28,70 & $-33,38$ & 74,28 \\
$\mathrm{~F}_{1}$ (L-005 x L-006) & $-20,59$ & 25,48 & $-36,52$ & 36,00 \\
$\mathrm{~F}_{1}$ (L-3436 x L-004) & $-8,77$ & 11,22 & $-17,83$ & 51,99 \\
$\mathrm{~F}_{1}$ (L-3436 x L-006) & $-8,25$ & 11,84 & $-17,69$ & 9,99 \\
$\mathrm{~F}_{1}$ (L-3509 x L-004) & $-9,59$ & 22,73 & $-26,25$ & 80,28 \\
$\mathrm{~F}_{1}$ (L-3509 x L-006) & $-12,47$ & 11,36 & $-20,97$ & 6,28 \\
$\mathrm{~F}_{1}$ (L-3513 x L-004) & $-6,41$ & 18,38 & $-20,97$ & 83,71 \\
$\mathrm{~F}_{1}$ (L-3513 x L-006) & $-9,70$ & 15,17 & $-21,68$ & 43,14 \\
$\mathrm{~F}_{1}$ (Magda x L-004) & $-17,80$ & 4,09 & $-20,97$ & $-13,43$ \\
$\mathrm{~F}_{1}$ (Magda x L-006) & $-16,86$ & 5,72 & $-21,26$ & $-4,86$ \\
$\mathrm{~F}_{1}$ (PIX-021E0818 x L-004) & $-12,04$ & 3,84 & $-15,26$ & $-19,15$ \\
$\mathrm{~F}_{1}$ (PIX-021E0818 x L-006) & $-15,88$ & 7,14 & $-21,40$ & $-2,57$ \\
$\mathrm{~F}_{1}$ (PIX-021E1235 x L-004) & $-19,24$ & 9,11 & $-25,96$ & 4,29 \\
$\mathrm{~F}_{1}$ (PIX-021E1235 x L-006) & $-22,74$ & 4,42 & $-26,11$ & 8,00 \\
$\mathrm{~F}_{1}$ (PIX-021E1545 x L-004) & $-15,58$ & 2,21 & $-17,26$ & 16,85 \\
$\mathrm{~F}_{1}$ (PIX-021E1545 x L-006) & $-16,92$ & 1,47 & $-17,97$ & $-0,29$ \\
$\mathrm{~F}_{1}$ (PIX-022E31 x L-004) & $-15,86$ & 8,82 & $-22,68$ & 36,28 \\
$\mathrm{~F}_{1}$ (PIX-022E31 x L-006) & $-13,12$ & 7,03 & $-18,69$ & 7,42 \\
$\mathrm{~F}_{1}$ (PIX-023D09 x L-004) & $-12,60$ & $-3,77$ & $-9,13$ & 4,29 \\
$\mathrm{~F}_{1}$ (PIX-023D09 x L-006) & $-11,47$ & $-10,41$ & $-1,14$ &
\end{tabular}

\section{CONCLUSÕES}

- Os híbridos experimentais apresentaram heterose para diâmetro de fruto e profundidade de inserção do pedúnculo;

- A linhagem parental do grupo II L-004 apresentou maior valor positivo de CGC para comprimento de fruto, diâmetro de fruto e profundidade de inserção do pedúnculo, enquanto a linhagem L-006 apresentou maior valor positivo de CGC para relação comprimento/diâmetro de fruto;

- A linhagem L-004 possui, predominantemente, alelos recessivos para a característica profundidade de inserção de pedúnculo;
- As linhagens L-004 e L-006 possuem freqüência semelhante de alelos dominantes para as características comprimento de fruto, diâmetro de fruto e relação comprimento/diâmetro;

- A característica profundidade de inserção do pedúnculo é condicionada, predominantemente, por alelos recessivos, com a dominância no sentido de diminuir sua média nos híbridos;

- Os melhores híbridos, com perspectiva para uso comercial, para a maioria das características avaliadas foram L-3436 x L-004, L-3513 x L-004 e L-3509 x L-004; 


\section{AGRADECIMENTOS}

Ao Conselho Nacional de Desenvolvimento Científico e Tecnológico (CNPq) pela concessão da bolsa de estudos e a HortiAgro Sementes, pelo apoio na realização dos experimentos.

\section{REFERÊNCIAS BIBLIOGRÁFICAS}

BLANK, A. F. Teste precoce da capacidade combinatória de linhagens de pimentão(Capsicum annuum L.). 1997. $71 \mathrm{f}$. Tese (Doutorado em Fitotecnia) - Universidade Federal de Lavras, Lavras, 1997.

BLAT, A. F. Obtenção e avaliação de híbridos duplos de pimentão (capsicum annuum L.). 1999. 74 f. Dissertação (Mestrado em Genética e Melhoramento de Plantas) Universidade do Estado de São Paulo, Jaboticabal, 1999.

DESHPANDE, A. A.; ANAND, N.; RAMACHANDER, P. R. Ideotype differentiation of horticultural groups in Capsicum spp. Genética Agraria, [S.1.], v. 42, n. 4, p. 357 364, 1988.

ECHER, M. M. Reação de pimentão (Capsicum annuum L.) a Phytophthora capsici e Potato vírus Y (PVYm). 2001. $62 \mathrm{f}$. Tese (Doutorado em Fitotecnia) - Escola Superior de Agricultura Luís de Queiroz, Piracicaba, 2001.

GALVÊAS, P. A. O. Características agronômicas de sete cultivares de pimentão (Capsicum annuum $L$.) e heterose de seus híbridos. 1988. 83 f. Dissertação (Mestrado em Fitotecnia) - Universidade Federal de Viçosa, Viçosa, 1988.

IKUTA, H. Ensaio de híbridos F1, F2 e variedades resistentes a vírus de pimentão (Capsicum annuиm L.). Revista de Olericultura, Campinas, v. 11, p. 64, 1971

INNECCO, R. Avaliação do potencial agronômico de híbridos e capacidade combinatória de linhagens de pimentão (Capsicum annuum L.). 1995. 113 f. Tese (Doutorado em Fitotecnia) - Universidade Federal de Lavras, Lavras, 1995.

MELO, A. M. T. Análise genética de caracteres de fruto em híbridos de pimentão. 1997. 112 f. Tese (Doutorado em Genética e Melhoramento de Plantas) - Escola Superior de Agricultura “Luiz de Queiroz”, Universidade de São Paulo, Piracicaba, 1997.

MIRANDA, J. E. C. de. Análise genética de um cruzamento dialélico em pimentão (Capsicum annuum L.). 1987. 159 f. Tese (Doutorado em Genética e Melhoramento de Plantas) - Escola Superior de Agricultura "Luiz de Queiroz", Universidade de São Paulo, Piracicaba, 1987.

TAVARES, M. Heterose e estimativa de parâmetros genéticos em um cruzamento dialélico de pimentão (Capsicum annuum L.). 1993. 87 f. Dissertação (Mestrado em Genética e Melhoramento de Plantas) - Universidade Federal de Lavras, Lavras, 1993. 